\title{
JORDAN ALGEBRAS AND LIE ALGEBRAS OF TYPE $\mathrm{D}_{4}$
}

BY H. P. ALLEN ${ }^{1,2}$

Communicated by George D. Mostow, August 16, 1965

The existence of exceptional Lie algebras of type $\mathrm{D}_{4}$ has been known for many years. Comparatively recently, Jacobson gave a construction which produces the only known examples of exceptional $\mathrm{D}_{4}$ 's. Relatively little was known about these algebras and our main aim in this research is to study a broad class of $D_{4}$ 's, called Jordan $\mathrm{D}_{4}$ 's, which includes the known exceptional examples. We approach the questions of characterizing these algebras and determining isomorphism conditions between them through the use of Galois cohomology, where the Steinberg types are taken as the standards of comparison. In what follows, unless specific mention is made, all fields are assumed to have characteristic 0 or $p \geqq 5$.

Let $\Im$ be an exceptional central simple Jordan algebra over a field $P$ and let $\mathfrak{t}$ be a cubic semisimple associative subalgebra. Then $\mathfrak{D}(\Im / \mathfrak{t})$, the subalgebra of the derivation algebra of $\Im$ which maps $\mathfrak{l}$ onto 0 , is a Lie algebra of type $D_{4}$. If $\mathfrak{l}$ is a field, then $\mathfrak{D}(\mathfrak{I} / \mathfrak{l})$ is an exceptional $\mathrm{D}_{4}$ (these are Jacobson's examples). $\mathrm{D}_{4}$ 's of this sort are called Jordan $D_{4}$ 's. If $\Im=\mathfrak{h}\left(\mathfrak{G}_{3}, \gamma\right)$ is a split algebra then $\mathfrak{D}(\mathfrak{S} / \mathfrak{l})$ is a Steinberg type, and if $\Im=\mathfrak{h}\left(\mathfrak{S}_{3}, \gamma\right)$ is split while $\mathfrak{l}=\Sigma P e_{i}$ is a diagonal algebra, then $\mathfrak{D}\left(\Im / \Sigma P e_{i}\right)$ is the split $\mathrm{D}_{4}$ over $P$ (of classical type if char $P \neq 0$ ). We first determine $\operatorname{Aut}_{P} \mathfrak{D}$ and Aut $_{\Phi} \mathfrak{D}$ where $\Phi$ is a subfield of finite codimension and Galois in $P$.

Let $\Gamma L_{\Phi}\left(\Im / \Sigma P e_{i}\right)$ be the group of all $s$-semilinear transformations $(s \in G(P / \Phi))$ of $\Im$ which leave $\Sigma P e_{i}$ invariant and satisfy $N(X C)$ $=\rho N(X)^{s}$, where $\rho \in P^{*}$ and $N(X)$ is the generic norm form on $\Im$. If $C \in \Gamma L_{\Phi}\left(\Im / \Sigma P e_{i}\right)$ then " $D \rightarrow C^{-1} D C$ " $\in A u t_{\Phi} D$ and the correspondence $C \rightarrow$ " $D \rightarrow C^{-1} D C$ " is an epimorphism. The kernel which we denote by $K$ is isomorphic to $P^{*} \times P^{*} \times P^{*}$. If we let GL( $\left(S / \Sigma P e_{i}\right)$ be the subgroup of all $P$-linear transformations in $\Gamma L_{\Phi}\left(\Im / \Sigma P e_{i}\right)$, then

$$
I \rightarrow K \rightarrow G L\left(\Im / \Sigma P e_{i}\right) \rightarrow \operatorname{Aut}_{P} \mathfrak{D} \rightarrow I
$$

is exact. (1) is not suitable for an application of Galois cohomology

1 The research was supported by National Science Foundation grant no. NSF GP-4017.

2 These results are contained in a dissertation which was submitted to Yale University in 1965 and was conducted under the supervision of Professor N. Jacobson. 
since $K$ is not in the center of $\mathrm{GL}\left(\Im / \Sigma P e_{i}\right)$. However we may circumvent this obstruction as follows.

Each $C \in \Gamma L_{\Phi}\left(\Im / \Sigma P e_{i}\right)$ affects a permutation of $\left\{P e_{1}, P e_{2}, P e_{3}\right\}$ say $P(C)$, and we let $\mathrm{GL}\left(\Im /\left\{P e_{i}\right\}_{i}\right)$ be the subgroup of $\mathrm{GL}\left(\Im / \Sigma P e_{i}\right)$ with $p(C)=1$. If Aut $_{P}^{\prime} \mathfrak{D}$ is the subgroup of Aut $_{P} \mathfrak{D}$ corresponding to $\mathrm{GL}\left(\Im /\left\{P e_{i}\right\}_{i}\right)$, then

$$
I \rightarrow K \rightarrow G L\left(\Im /\left\{P e_{i}\right\}_{i}\right) \rightarrow \operatorname{Aut}_{P}^{\prime} \mathfrak{D} \rightarrow I
$$

is exact and $K$ is in the center of GL( $\left.\Im /\left\{P e_{i}\right\}_{i}\right)$.

Now let $\mathfrak{R}$ be a $\Phi$-form of $\mathfrak{D}=\mathfrak{D}\left(\Im / \Sigma P e_{i}\right), \Im$ split, and let $r \rightarrow \eta(r)$ be the corresponding pre-cocycle. Recall that $r \rightarrow \eta(r)$ is a homomorphism of $G=G(P / \Phi)$ into Aut ${ }_{\Phi} \mathfrak{D}$ such that $\eta(r)$ is $r$-semilinear, and that $\mathbb{R}$ is the set of fixed points of $\mathfrak{D}$ under every $\eta(r)$. If $\eta(r)$ is conjugation by $C_{r} \in \Gamma L_{\Phi}\left(\Im / \Sigma P e_{i}\right)$, we let $p(r)$ be the permutation of $\left\{P e_{1}, P e_{2}, P e_{3}\right\}$ induced by $C_{r}$. Then $r \rightarrow p(r)$ is a homomorphism of $G$ into $S_{3}$ and the order of $p(G)$ is called the $D_{4}$ type of $\&$ and is denoted by roman numeral subscripts, e.g.,

$$
\mathrm{D}_{4 \mathrm{I}}, \mathrm{D}_{4 \mathrm{II}}, \mathrm{D}_{4 \mathrm{III}} \text {, and } \mathrm{D}_{4 \mathrm{VI}} \text {. }
$$

(The exceptional algebras are those of type $\mathrm{D}_{4 \mathrm{III}}$ and $\mathrm{D}_{4 \mathrm{VI}}$.)

It is easy to see that there are only a finite number of Steinberg $\mathrm{D}_{4}$ 's over $\Phi$ which are split by $P$, and more importantly, if $\eta$ is any pre-cocycle of $G$ in $\operatorname{Aut}_{\Phi} \mathfrak{D}$, then there is a pre-cocycle $\mu$ corresponding to a Steinberg $D_{4}$, such that $\mu^{-1} \eta$ has values in $\operatorname{Aut}_{P}^{\prime} \mathfrak{D}$. If we use such an $\mu$ to equip $\operatorname{Aut}_{P} \mathfrak{D}$ with a $G$-group structure, then Aut $_{P}^{\prime} \mathfrak{D}$ is a $G$-subgroup and we may define a $G$-action on each of the groups in (2) so that (2) becomes an exact sequence of $G$-groups and $G$-homomorphisms. The preceding remarks show that relative to this action, the 1 -cocycle of $G$ in $\operatorname{Aut}_{P} \mathfrak{D}$ corresponding to $\mathfrak{R}$ has values in Aut $_{P}^{\prime} \mathfrak{D}$.

From (2) we obtain the exact sequence of first cohomology sets

$$
\begin{aligned}
& I=H_{\mu}^{1}(G, K) \rightarrow H_{\mu}^{1}\left(G, G L\left(\Im /\left\{P e_{i}\right\}_{i}\right)\right) \rightarrow H_{\mu}^{1}\left(G, \operatorname{Aut}_{P}^{\prime} \mathfrak{D}\right) \\
& \quad \stackrel{\Delta}{\rightarrow} H_{\mu}^{2}(G, K)
\end{aligned}
$$

where the subscript $\mu$ is used to indicate the generation of the $G$ action and $\Delta$ is the coboundary operator. If $\mathbb{R}$ is an arbitrary $\Phi$-form of $\mathfrak{D}$, let $[\gamma]_{\mu}$ be its corresponding 1 -cocycle in the appropriate $H_{\mu}^{1}\left(G, \operatorname{Aut}_{P}^{\prime} \mathfrak{D}\right)$ as above. We set $\Gamma(\Omega)=[\gamma]_{\mu} \Delta \in H_{\mu}^{2}(G, K)$ and call it the 2-cohomology class of $\mathbb{R}$.

The sets in (3) have simple interpretations: the elements of $H_{\mu}^{1}\left(G, \mathrm{GL}\left(\Im /\left\{P e_{i}\right\}_{i}\right)\right)$ correspond to certain norm equivalence classes 
of $\Phi$-forms of isotopes $\Im^{(u)}$ of $\Im$ where $u \in \Sigma P e_{i}$, whereas the elements of $H_{\mu}^{2}(G, K)$ correspond to certain classes of separable associative algebras over $\Phi$ which are split by $P$, and play a role which generalizes the relationship between central simple associative algebras and factor sets. Finally if $B$ is the associative subalgebra of $\operatorname{Hom}_{P}(\Im, \Im)$ strictly generated by $\mathbb{R}$ over $\Phi$, then $(B)$ is a $\Phi$-form of $\left(P_{8} \oplus P_{8} \oplus P_{8}, J\right)$ where $J$ is an involution whose restriction to each $P_{8}$ is the involution determined by the norm form on $\mathbb{E}$. The 2-cohomology class of $\mathbb{R}$, $\Gamma(\mathfrak{R})$, is then the class corresponding to $(\$)$ in $H_{\mu}^{2}(G, K)$.

We now have the following results.

THEOREM 1. Let $\mathfrak{R}$ be a $\Phi$-form of $\mathfrak{D}=\mathfrak{D}\left(\Im / \Sigma P e_{i}\right)$ where $P / \Phi$ is a finite dimensional Galois extension. Then $\mathfrak{R}$ is a Jordan $\mathrm{D}_{4}$ if and only if $\Gamma(\Omega)=1$.

The condition $\Gamma(\mathbb{R})=1$ is equivalent to the assertion that if $\&$ is the $\Phi$-enveloping algebra of $\mathfrak{l}$, as above, then every simple component of $(5)$ is a total matrix algebra over its center.

Theorem 2. A Lie algebra of type $\mathrm{D}_{4}$ over a field $\Phi$ of characteristic 0 is a Jordan $\mathrm{D}_{4}$ if and only if it is a subalgebra of an algebra of type $F_{4}$.

Theorem 2 follows readily from the conjugacy of subalgebras of type $D_{4}$ of a Lie algebra of type $F_{4}$, over an algebraically closed field of characteristic 0.

THEOREM 3. Let $\mathfrak{R}=\mathfrak{D}(\Im / \mathfrak{l})$ and $\mathfrak{R}^{1}=\mathfrak{D}\left(\Im^{1} / \mathfrak{l}^{1}\right)$ be Jordan $\mathrm{D}_{4}$ 's. Then

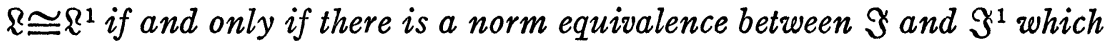
carries $\mathfrak{l}$ onto $\mathfrak{l}^{1}$.

Using these results we obtain

THEOREM 4. If $\Phi$ is a local or finite field then there is a 1-1 correspondence between the set of Jordan $\mathrm{D}_{4}$ 's over $\Phi$ and the set of overfields of $\Phi$ of degree at most 3. In particular, every exceptional $\mathrm{D}_{4}$ over $\Phi$ is a Jordan $\mathrm{D}_{4}$.

Globally we can prove the following:

TheOREM 5. Let $\Phi$ be a global field, \& a Lie algebra of type $\mathrm{D}_{4}$ over $\Phi$. Then $\&$ is a Jordan $\mathrm{D}_{4}$ if and only if $\mathfrak{R}_{\Phi_{p}}$ is a Jordan $\mathrm{D}_{4}$ for every prime $p$ on $\Phi$.

THEOREM 6. Let $\Phi$ and $\mathfrak{R}$ be as in the statement of Theorem 5. Then the number of primes $p$ on $\Phi$ such that $\mathfrak{R}_{\Phi_{p}}$ is not a Jordan $\mathrm{D}_{4}$ is finite: moreover none of these is real.

YALE UNIVERSITY 